\title{
Bianchi Type VI0 Inflationary Universe with Constant Deceleration Parameter and Flat Potential in General Relativity
}

\author{
Raj Bali and Parmit Kumari \\ Department of Mathematics, University of Rajasthan, Jaipur-302004, India \\ Email: balir5@yahoo.co.in
}

\begin{abstract}
Inflationary universe scenario with constant deceleration parameter in the presence of massless scalar field and flat potential taking Bianchi Type $\mathrm{VI}_{0}$ space time as a source is discussed. We find that the rate of expansion slows down with increase of time. It is also observed that the ratio of shear and expansion is non-zero for all values of $\mathrm{T}$ where $\mathrm{at}+\mathrm{b}=\mathrm{T}$, $\mathrm{t}$ being cosmic time, $\mathrm{a}$ and $\mathrm{b}$ being constants. Thus the universe remains anisotropic throughout the evolution. The Higgs field is constant for large values of $\mathrm{T}$ when $\alpha<2$ and the Higgs field evolves slowly but the universe expands for $\alpha>2$ where $\alpha$ is a constant. It may be positive and negative both. The model represents decelerating and accelerating phases of universe and has Point Type singularity at $\mathrm{T}=0$ (MacCallum [25]). In special case i.e. if $\mathrm{N}=0$ and $\alpha>0$ then the model isotropizes at late time, $\mathrm{N}$ being constant of integration.
\end{abstract}

Keywords: Bianchi $\mathrm{VI}_{0}$, Inflationary, deceleration parameter, flat potential

\section{Introduction}

The primordial acceleration in which the universe undergoes rapid exponential expansion is known as inflation. The notion of inflation is so far one of the best mechanism at the early stages of evolution of the universe to explain the flat, homogeneous and isotropic nature of the present day universe. In these models, the universe undergoes a phase transition characterized by the evolution of Higgs field $\varphi$. The inflation will take place if potential $\mathrm{V}(\varphi)$ has flat region and in this region, the Higgs field $\varphi$ evolves slowly but the universe expands in an exponential way due to vacuum field energy. The flat part of the potential is naturally associated with a vacuum energy that is identified as an effective cosmological constant $(\Lambda)$ which makes the universe enter an inflationary period. Historically, a model closely related to the inflationary universe was suggested by Starobinsky [1] but the inflationary cosmological models became popular after an important paper of Guth [2] who proposed inflationary model in the context of grand unified field theory (GUT). There are two basic types of inflationary models: one is due to appearance of a flat potential e.g. in GUT's phase transitions and the other is due to a scalar curvaturesquarred term. Barrow and Turner [3] pointed out that a universe with a large amount of anisotropy will not undergo the inflationary phase. A universe with only moderate anisotropy will undergo inflation and will be rapidly isotropized. Sato [4] in a study has shown that the most prevailing inflationary models are investigated through the scalar field which acts as a source of inflation and generates cosmic acceleration. Inflationary scenario for homogeneous and isotropic models (FRW models) has been studied by many authors viz. Linde [5], Wald [6], Barrow [7], Abrecht and Steinhardt [8], Abbott and Wise [9], La and Steinhardt [10], Mataresse and Lucchin [11]. Rothman and Ellis [12] have pointed out that we can have solution for isotropic problem if we work with anisotropic metric but isotropize in special cases. In view of these observations Singh [13] investigated Bianchi Type II inflationary models with constant deceleration parameter in general relativity. Bali [14] investigated inflationary scenario in Bianchi Type I space time with flat potential.

The universe in smaller scale is neither homogeneous nor isotropic nor do we expect the universe to have these properties in its early stages. Also Astronomical observations in late eighties revealed that the predictions of FRW models do not always meet our requirements as were believed earlier (Smoot et. al. [15]). Therefore, spatially homogeneous and anisotropic Bianchi space times (I-IX) are considered to study the universe in its early stages of evolution. Among these, Bianchi Type $\mathrm{VI}_{0}$ space time is of 
particular interest because this is simple generalization of Bianchi Type I space time. Barrow [16] pointed out that Bianchi Type $\mathrm{VI}_{0}$ cosmological models give a better explanation of some of the cosmological problems like helium abundance and these can be istropized in special cases. Seeing the importance of these models, various authors viz. Ellis and MacCallum [17], Collins [18], Roy and Singh [19], Tikekar and Patel [20], Bali et. al. [21,22], Ram and Singh [23] have investigated cosmological models considering Bianchi Type $\mathrm{VI}_{0}$ space - time in different contexts.

\section{$2 \quad$ Metric and Field Equations}

We consider Bianchi Type $\mathrm{VI}_{0}$ metric in the form

$$
\mathrm{ds}^{2}=-\mathrm{dt}^{2}+\mathrm{A}^{2} \mathrm{dx}^{2}+\mathrm{B}^{2} e^{2 \mathrm{x}} \mathrm{dy}^{2}+\mathrm{C}^{2} \mathrm{e}^{-2 \mathrm{x}} d z^{2}
$$

where $\mathrm{A}, \mathrm{B}, \mathrm{C}$ are metric potentials and functions of t-alone.

We assume the co-ordinates to be comoving so that

$$
\mathrm{v}^{1}=0=\mathrm{v}^{2}=\mathrm{v}^{3}, \mathrm{v}^{4}=1
$$

The action of the gravitational field minimally coupled to a scalar field with potential $\mathrm{V}(\varphi)$ is given by Stein-Schabes [24] as

$$
\mathrm{S}=\int \sqrt{-\mathrm{g}}\left[\mathrm{R}-\frac{1}{2} \mathrm{~g}^{\mathrm{ij}} \partial_{\mathrm{i}} \phi \partial_{\mathrm{j}} \phi-\mathrm{V}(\phi)\right] \mathrm{d}^{4} \mathrm{x}
$$

The Einstein's Field equations (in gravitational units $\mathrm{G}=\mathrm{c}=1$ ) in the case of massless scalar field $\varphi$ with potential $\mathrm{V}(\phi)$ are given by

$$
\mathrm{R}_{\mathrm{ij}}-\frac{1}{2} R \mathrm{~g}_{\mathrm{ij}}=-8 \pi \mathrm{T}_{\mathrm{ij}}
$$

with

$$
\mathrm{T}_{\mathrm{ij}}=\partial_{\mathrm{i}} \phi \partial_{\mathrm{j}} \phi-\left[\frac{1}{2} \partial_{\rho} \phi \partial^{\rho} \phi+\mathrm{V}(\phi)\right] \mathrm{g}_{\mathrm{ij}}
$$

The conservation relation $\mathrm{T}_{\mathrm{i} ; \mathrm{j}}^{\mathrm{j}}=0$ leads to

$$
\frac{1}{\sqrt{-g}} \partial_{\mu}\left(\sqrt{-g} \partial_{\mu} \phi\right)=-\frac{d V}{d \phi}
$$

The Einstein's Field equation (3) for the metric (1) leads to

$$
\begin{gathered}
\frac{B_{4} C_{4}}{B C}+\frac{B_{44}}{B}+\frac{C_{44}}{C}+\frac{1}{A^{2}}=-8 \pi\left[\frac{1}{2} \phi_{4}^{2}-V(\phi)\right] \\
\frac{A_{44}}{A}+\frac{A_{4} C_{4}}{A C}+\frac{C_{44}}{C}-\frac{1}{A^{2}}=-8 \pi\left[\frac{1}{2} \phi_{4}^{2}-V(\phi)\right] \\
\frac{A_{44}}{A}+\frac{A_{4} B}{A B}+\frac{B_{44}}{B}-\frac{1}{A^{2}}=-8 \pi\left[\frac{1}{2} \phi_{4}^{2}-V(\phi)\right] \\
\frac{A_{4} B_{4}}{A B}+\frac{A_{4} C_{4}}{A C}+\frac{B_{4} C_{4}}{B C}-\frac{1}{A^{2}}=8 \pi\left[\frac{1}{2} \phi_{4}^{2}+V(\phi)\right] \\
\frac{B_{4}}{B}-\frac{C_{4}}{C}=0
\end{gathered}
$$

Equation (10) leads to

$$
B=m C
$$

where $\mathrm{m}$ is constant of integration.

\section{Solution of Field Equation}

The equation (5) for the scalar field $(\varphi)$ leads to 


$$
\phi_{44}+\left(\frac{A_{4}}{A}+\frac{2 C_{4}}{C}\right) \phi_{4}=-\frac{d V}{d \phi}
$$

where suffix ' 4 ' indicates ordinary partial derivatives with respect to t.

We are interested in inflationary solution so flat region is considered. Thus $\mathrm{V}(\phi)=\mathrm{K}$ is constant.

Now equation (12) leads to

$$
\phi_{44}+\left(\frac{A_{4}}{A}+\frac{2 C_{4}}{C}\right) \phi_{4}=0
$$

From equation (13), we have

$$
\phi_{4}=\frac{\ell}{A C^{2}}
$$

where $\ell$ is constant of integration.

The scale factor $\mathrm{R}$ for line-element (1) is given by

$$
R^{3}=A B C=m A C^{2}
$$

To find the deterministic model of the universe, we assume that deceleration parameter is constant. The deceleration parameter in Cosmology is a dimensionless measure of the cosmic acceleration of the expansion of space in FRW universe. It is defined as

$$
q=\frac{-R_{44} / R}{R_{4}^{2} / R^{2}}=\alpha(\text { Constant })
$$

where $\alpha>0$ or $\alpha<0$ and $\mathrm{R}$ is scale factor of the universe. If constant deceleration is taken then accelerating and decelerating phases are included in the following manner. The expansion of the universe is said to be accelerating if $R_{44}>0$ and in this case, $q<0$ as per recent astronomical observations.

Observations of the cosmic microwave background demonstrate that the universe is very nearly flat, so $\mathrm{q}>0$.

This implies that the universe is decelerating. However, observations of distant $I_{a}$ Supernovae indicate that $\mathrm{q}<0$ and the expansion of universe is accelerating. Thus, it is interesting to assume that decelerating parameter is constant with the help of which we can explain the decelerating and accelerating phases of universe.

From equation (16), we have

$$
\frac{R_{44}}{R_{4}}+\alpha \frac{R_{4}}{R}=0
$$

which leads to

$$
R=(a t+b)^{\frac{1}{\alpha+1}}
$$

where $\mathrm{a}=\beta(\alpha+1), \beta$ is constant of integration.

From equation (15) and (17), we have

$$
A C^{2}=\frac{1}{m}(a t+b)^{\frac{3}{\alpha+1}}
$$

Adding equation (6) and (9), we have

$$
\frac{C_{44}}{C}+\frac{C_{4}^{2}}{C^{2}}+\frac{A_{4} C_{4}}{A C}=8 \pi K
$$

Multiplying equation (19) by $A C^{2}$, we have

$$
A C C_{44}+A C_{4}^{2}+A_{4} C_{4} C=8 \pi K A C^{2}
$$

After using (18), we have

$$
\left(A C C_{4}\right)_{4}=\frac{8 \pi K}{m}(a t+b)^{\frac{3}{\alpha+1}}
$$

Thus, we have 


$$
A C C_{4}=\frac{8 \pi K(\alpha+1)}{m a(\alpha+4)}(a t+b)^{\frac{\alpha+4}{\alpha+1}}+N
$$

where $\mathrm{N}$ is constant of integration.

Dividing equation (22) by $\mathrm{A} \mathrm{C}^{2}$ and using equation (18), we have

$$
\frac{C_{4}}{C}=\frac{8 \pi K(\alpha+1)}{a(\alpha+4)}(a t+b)+m N(a t+b)^{-\frac{3}{\alpha+1}}
$$

Equation (23) leads to

$$
\log C=\frac{8 \pi K(\alpha+1)}{2 a^{2}(\alpha+4)}(a t+b)^{2}+\frac{m N(\alpha+1)}{a(\alpha-2)}(a t+b)^{\frac{\alpha-2}{\alpha+1}}+\log L
$$

which leads to

$$
C=L \exp \left(\gamma T^{2}+\lambda T^{\frac{\alpha-2}{\alpha+1}}\right)
$$

where

$$
\gamma=\frac{8 \pi K(\alpha+1)}{2 a^{2}(\alpha+4)}, \lambda=\frac{m N(\alpha+1)}{a(\alpha-2)}
$$

and $\mathrm{L}$ is constant of integration.

Now

$$
B=m C=m L \exp \left(\gamma T^{2}+\lambda T^{\frac{\alpha-2}{\alpha+1}}\right)
$$

From equation (18), we have

$$
A=\frac{T^{\frac{3}{\alpha+1}}}{m L^{2}} e^{-2\left(\gamma T^{2}+\lambda T^{\frac{\alpha-2}{\alpha+1}}\right)}
$$

where $\mathrm{at}+\mathrm{b}=\mathrm{T}$.

After suitable transformation of coordinates, the metric (1) leads to the form

$$
d s^{2}=-\frac{1}{a^{2}} d T^{2}+\frac{T^{\frac{6}{\alpha+1}}}{\left.e^{4\left(\gamma T^{2}+\lambda T^{\frac{\alpha-2}{\alpha+1}}\right.}\right)} d X^{2}+e^{2\left(\gamma T^{2}+\lambda T^{\frac{\alpha-2}{\alpha+1}}\right)}\left\{e^{2 m L^{2} X} d Y^{2}+e^{-2 m L^{2} X} d Z^{2}\right\}
$$

where

$$
\frac{1}{m L^{2}} x=X, m L y=Y, L z=Z
$$

\section{$4 \quad$ Physical and Geometrical Features}

The rate of Higgs fields $(\phi)$ is given by equation (14) as

which leads to

$$
\phi_{4}=\frac{\ell}{A C^{2}}=\frac{\ell m}{T^{\frac{3}{\alpha+1}}}
$$

$$
\phi=\frac{\ell m(\alpha+1)}{a(\alpha-2)} T^{\frac{\alpha-2}{\alpha+1}}+M
$$

where $\mathrm{M}$ is constant of integration.

The spatial volume $\left(\mathrm{R}^{3}\right)$, the Hubble parameter $(\mathrm{H})$, the expansion $(\theta)$ and shear $(\sigma)$ for the model (27) are given by 


$$
\begin{gathered}
R^{3}=m A C^{2}=T^{\frac{3}{\alpha+1}} \\
H=\frac{a}{(\alpha+1) T} \\
3 H=\frac{3 a}{(\alpha+1) T} \\
\sigma=\frac{1}{\sqrt{3}}\left|\frac{A_{4}}{A}-\frac{B_{4}}{B}\right|=\frac{3 a}{(\alpha+1) T}-6 a \lambda T-\frac{3 a \lambda(\alpha-2)}{(\alpha+1)} T^{-\frac{3}{\alpha+1}}
\end{gathered}
$$

\section{Conclusion}

The spatial volume increases with time. The rate of expansion slows down with the increase of time and finally drops to zero when $\mathrm{T} \rightarrow \infty$. It is also observed that the ratio of shear and expansion is non-zero for all values of T. Thus universe remains anisotropic throughout the evolution and the Higgs field $(\phi)$ is constant for large value of $\mathrm{T}$ when $\alpha<2$. When $\alpha>2$ then the Higgs field $(\phi)$ evolves slowly but the universe expands. Thus the model represents shearing, non-rotating and expanding universe. Also since deceleration parameter $\mathrm{q}=\alpha$ (constant). Therefore, the model represents decelerating and accelerating phases of universe when $\alpha>0$ and $\alpha<0$ respectively and the model (27) indicates the results as per astronomical observations. At the time of evolution of universe, the anisotropy is constant and during the inflation, it is still homogeneous and anisotropic. Thus the results obtained in this paper can be interpreted in the framework of perfect fluid scenario. The model (27) has Point Type singularity at $\mathrm{T}$ $=0$ (MacCallum[25]). During an initial period, the universe is assumed to be dominated by constant potential term $\mathrm{V}(\phi)$ with scalar field $\phi$ giving rise to power law inflation. Hence inflationary scenario is observed in Bianchi Type $\mathrm{VI}_{0}$ space time in presence of massless scalar field with flat potential.

Acknowledgments. The authors are thankful to the Referee for his valuable comments and suggestions.

\section{References}

1. A.A. Starobinsky, "A new type of isotropic cosmological models without singularity," Phys. Lett. B, vol.91, pp. 99-102, 1980.

2. A.H. Guth, "Inflationary universe: A possible solution to the horizon and flatness problems," Phys. Rev. D, vol.23, pp. 347-356, 1981.

3. D. Barrow and M.S. Turner, "Inflation in the universe," Nature, vol.292, pp. 35-38, 1981.

4. K. Sato, "First order phase transition of vacuum and expansion of the universe," Monthly Not. Roy. Astron. Soc. Vol.195, pp. 467-479, 1981a.

5. A.D. Linde, "A new inflationary universe scenario: A possible solution of the horizon, flatness, homogeneity, isotropy and primordial monopole problems," Phys. Lett. B, vol.108, pp. 389-393, 1982.

6. R.M. Wald, "Asymptotic behavior of homogeneous cosmological models in presence of a positive cosmological constant," Phys. Rev. D, vol.28, pp. 2118, 1983.

7. J.D. Barrow, "Cosmic no hair theorems and inflation ," Phys. Lett. B, vol.187, pp. 12-16, 1987.

8. A. Albrecht and P.J. Steinhardt, "Cosmology for grand unified theories with radiatively induced symmetry breaking," Phys. Rev. Lett., vol.48, pp. 1220-1223, 1982.

9. L.F. Abbott and M.B. Wise, "Constraints on generalized inflationary cosmologies," Nucl. Phys. B, vol.244, pp. 541-548, 1984 .

10. D. La and P.J. Steinhardt, "Extended inflationary cosmology," Phys. Rev. Lett., vol.62, pp. 376, 1989.

11. S. Matarrese and F. Lucchin, "Power law inflation," Phys. Rev. D, vol.32, pp. 1316-1322, 1985.

12. T. Rothman and G.F.R. Ellis, "Can inflation occur in anisotropic cosmologies," Phys. Lett. B, vol.180, pp. 19-24, 1986. 
13. C.P. Singh, "Bianchi Type II inflationary models with constant deceleration parameter in general relativity," Pramana-J of Phys., vol.68, pp. 707-720, 2007.

14. R. Bali, "Inflationary scenario in Bianchi Type I space-time," Int. J. Theor. Phys., vol. 50, pp. 3043-3048, 2011.

15. Smoot et. al., "Structure in the COBE differential microwave radiometer first year map," Astrophys. J. Part 2Letters 396, pp. L1-L5, 1992.

16. J.D. Barrow, "Helium formation in cosmologies with anisotropic curvature," Roy. Astron. Society, vol.211, pp. 108-141, 1984.

17. G.F.R. Ellis and M.A.H. MacCallum, "A class of homogeneous cosmological models," Comm. In Math. Phys., vol.12, pp. 108-141, 1969.

18. C.B. Collins, "More qualitative cosmology," Comm. in Math. Phys., vol.23, pp. 137-158, 1971.

19. S.R. Roy and J.P. Singh, "Some Bianchi Type $V I_{0}$ cosmological models with free gravitational field of magnetic type," Acta Physica Austriaca, vol.55, pp. 57-66, 1983.

20. R. Tikekar and L.K. Patel, "Some exact solutions in Bianchi $V I_{0}$ string cosmology," Pramana-J. Phys., vol.42, pp. 483-489, 1994.

21. R. Bali, R. Banerjee and S.K. Banerjee, "Some LRS Bianchi Type $V I_{0}$ cosmological models with special free gravitational fields," Electron. J. Theor. Phys., vol.6, pp. 165-174, 2009.

22. R. Bali, R. Banerjee and S.K. Banerjee, "Bianchi Type $V I_{0}$ bulk viscous massive string cosmological models in general relativity," Astrophys. Space-Sci., vol. 317, pp. 21-26, 2008.

23. S. Ram and P. Singh, "Bianchi Type $V I_{0}$ cosmological model in certain theories of gravitation," Astrophys. Space-Sci., vol.200, pp. 35-46, 1993.

24. J.A. Stein-Schabes, "Inflation in spherically symmetric inhomogeneous models," Phys. Rev. D., vol.35, pp. 2345$2351,1987$.

25. M.A.H. MacCallum, "A class of homogeneous cosmological models III, asymptotic behavior," Comm. in Math. Phys., vol.20, pp. 57-74, 1971. 